\title{
Protective Effects of Kaempferitrin on Advanced Glycation End Products Induce Mesangial Cell Apoptosis and Oxidative Stress
}

\author{
Wenxian Jiang ${ }^{1}$, Rongshen Wang ${ }^{1}{ }^{1}$, Di Liu ${ }^{1}$, Min Zuo ${ }^{1}$, Chunzhen Zhao ${ }^{1}$, Tianliang Zhang ${ }^{2}$ \\ and Wanzhong $\mathrm{Li}^{1, *}$ \\ 1 School of Pharmacy, Weifang Medical University, Weifang 261053, China; jiangwenxian123@163.com (W.J.); \\ wangrs199012@126.com (R.W.); ld0928928@163.com (D.L.); ZuoMin1026@163.com (M.Z.); \\ zhaochunzhen@wfmc.edu.cn (C.Z.) \\ 2 Experimental Center for Medical Research, Weifang Medical University, Weifang 261053, China; \\ zhangtl@wfmc.edu.cn \\ * Correspondence: liwz@wfmc.edu.cn; Tel.: +86-536-8462490
}

Received: 23 September 2018; Accepted: 17 October 2018; Published: 26 October 2018

\begin{abstract}
Advanced glycation end products (AGEs) and the receptor for AGEs (RAGE) both play important roles in diabetic nephropathy (DN). Previous studies have identified glomerular mesangial cells (GMCs) injury as a key early risk factor in the development of DN. Kaempferitrin (KM) is a potent antioxidant with hypoglycemic action. Although KM is known to protect against AGE-induced damage in GMCs, the effects and the mechanisms by which they occur are poorly understood. In this study, cultured rat GMCs were exposed to AGE-induced oxidative stress (OS) to model DN in vitro. Reactive oxygen species (ROS) was analyzed by $2^{\prime}, 7^{\prime}$-dichlorofluorescin diacetate (DCFH-DA). Superoxide dismutase (SOD) and malondialdehyde (MDA) were studied using commercial kits. Mitochondrial membrane potential $(\Delta \psi \mathrm{m})$ was measured by rhodamine 123 . Hoechst 33258 and annexin $\mathrm{V}$ and propidium iodide (PI) double staining were performed to observe the apoptosis states in GMCs, whereas apoptosis and protective mechanism in AGE-induced GMCs were investigated by Western blot. The data revealed that KM effectively increased SOD activity, decreased MDA levels, suppressed ROS generation, and protected against OS in AGE-induced GMCs. Treatment with KM also inhibited the expression of collagen IV and transforming growth factor- $\beta 1$ (TGF- $\beta 1$ ), improved mitochondrial membrane potential recovery, and suppressed the mitochondrial/cytochrome c-mediated apoptosis pathway through the expression of anti-apoptotic factors in GMCs in vitro. These findings suggest that KM may be a new potential agent in the treatment of DN in future.
\end{abstract}

Keywords: kaempferitrin; mesangial cells; advanced glycation end products; oxidative stress; apoptosis; signal pathway

\section{Introduction}

Diabetes mellitus (DM) is a chronic metabolic disorder associated with long-term damage and failure of various organs [1]. Diabetic nephropathy (DN) is a serious and common chronic microvascular complication of DM [2], that is the main cause of mortality in patients with diabetes, as well as a major contributor to the high prevalence of end-stage renal disease (ESRD) worldwide [3]. The global prevalence of DM is growing rapidly, especially in developing countries, and the incidence rates of both DN and ESRD continue to increase [4]. Hyperglycemia is accompanied by an accelerated rate of formation of advanced glycation end products (AGEs). AGEs, which are derived from reducing the non-enzymatical reaction of sugars with amino groups of protein, play an important role in 
the pathogenesis of diabetic complications [5]. Despite recent progress, the underlying molecular mechanisms behind DM-induced renal damage are still poorly understood.

AGEs and RAGE both play a role in the pathogenesis of DN [6], triggering signaling cascade events and causing damage through oxidative stress (OS) [7]. OS is the result of excessive production of reactive oxygen species (ROS) [8], which can induce mitochondrial dysfunction, decrease adenosine triphosphate (ATP) production, and lead to the development of DN [9]. The transforming growth factor beta (TGF- $\beta$ ) family proteins are heavily involved in the progression of OS by regulating the expression levels of associated proteins and antioxidant enzymes [10]. AGEs accumulate in the glomerular basement membrane and in mesangial cells [11]. GMCs are key contributors to glomerulosclerotic and renal lesions in DM [12]. AGEs can influence cell function and induce OS and apoptosis both by interacting with receptors and by reducing basement membrane deformability, degradation, and matrix cell interaction [13]. However, the mechanisms behind AGE-induced GMCs injury, and the protective agents involved in the reduction of renal damage, have not been thoroughly explored.

Flavonoids are widely distributed in numerous flowering and non-flowering plants and are known as a potential source for new drugs due to their chemical diversities and low side-effect rates and toxicities [14]. Flavonoids are potent antioxidants, free radical scavengers [15] with anti-aging [16], neuroprotective [17], hypoglycemic actions [18,19], and anti-inflammatory properties [20]. Kaempferitrin (kaempherol-3, 7-bisrhamnoside; KM) is a naturally occurring flavonoid glycoside from many plants [21] that possesses antimicrobial activity [22], antitumor effect [23], antioxidative [24], and hypoglycemic activities $[25,26]$. It is thought that the antioxidative properties of flavonoids are potentially due to the ability of enzymes to obstruct OS [27]. Although they play a key role in the protection against apoptosis and OS, the effects of KM on AGE-induced injury of GMCs have not been characterized.

The use of AGE-induced renal cells as a model to study the cellular and molecular mechanisms of DN is widespread [28,29]. It is thought that AGEs may trigger OS, thereby accelerating GMCs apoptosis. However, the link between OS and apoptosis remains unclear. Therefore, our study aims to investigate the effects of KM on apoptosis and OS in AGE-stimulated mesangial cell and the underlying mechanism in vitro.

\section{Results}

\subsection{KM Increases the Viability of AGE-Treated GMCs}

To determine the effect that KM has on the viability of AGE-treated GMCs, cells were treated with different concentrations of KM $(10,20,35,70,104,140,175$, and $210 \mu \mathrm{M})$ or AGEs $(160 \mu \mathrm{g} / \mathrm{mL})$ for $24 \mathrm{~h}$. The cell viability of GMCs treated with AGEs alone decreased as compared to that of the control group, (Figure $1 ; p<0.01)$. Strikingly, the addition of even low concentrations of KM $(10,20$, and $35 \mu \mathrm{M})$ to the AGE-treated GMCs rescued the AGE treatment phenotype, with the survival rate of cells treated with KM significantly increased as compared to that of the AGE-treated group $(p<0.01)$. KM at concentrations of 10,20 , and $35 \mu \mathrm{M}$ were used for further experiments. These data show that $\mathrm{KM}$, even at low concentrations, has a protective effect against AGE-induced decreases in GMC viability. 


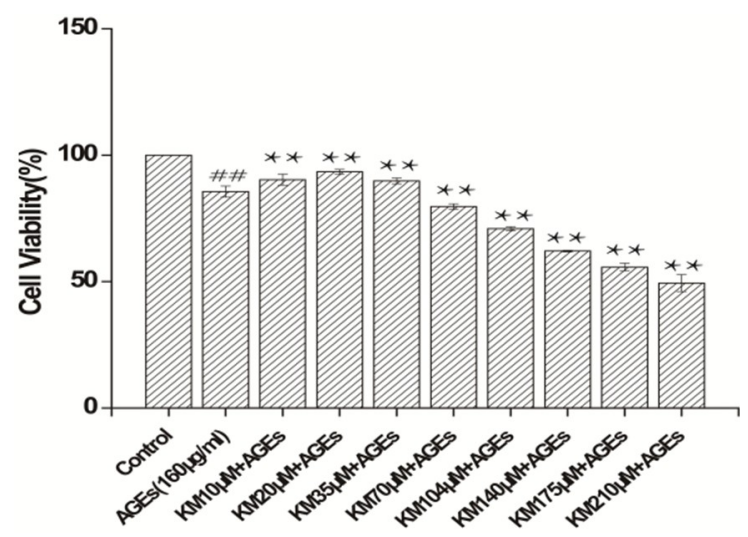

Figure 1. Kaempferitrin (KM) increases the cell viability of advanced glycation end (AGE)-treated glomerular mesangial cells (GMCs). GMCs were pre-treated with different concentrations of KM and then stimulated with AGEs $(160 \mu \mathrm{g} / \mathrm{mL})$ for $24 \mathrm{~h}$. The results are presented as mean \pm standard errors of the means (SEM). ${ }^{* *} p<0.01$, vs. AGEs group; ${ }^{\# \#} p<0.01$, vs. control group.

\subsection{KM Treatment Protects against AGE-Induced GMC Injury}

Cells were pre-treated with 10, 20, or $35 \mu \mathrm{M} \mathrm{KM}$ for $24 \mathrm{~h}$ and then incubated with AGEs for $24 \mathrm{~h}$. KM treatment ameliorated AGE-induced cell shrinkage and reduced the number of apoptotic vacuoles (Figure 2). The medium dose group $(20 \mu \mathrm{M})$ recovery was more obvious after cell injury. These data indicate that KM treatment protects against AGE-induced injury of GMCs.

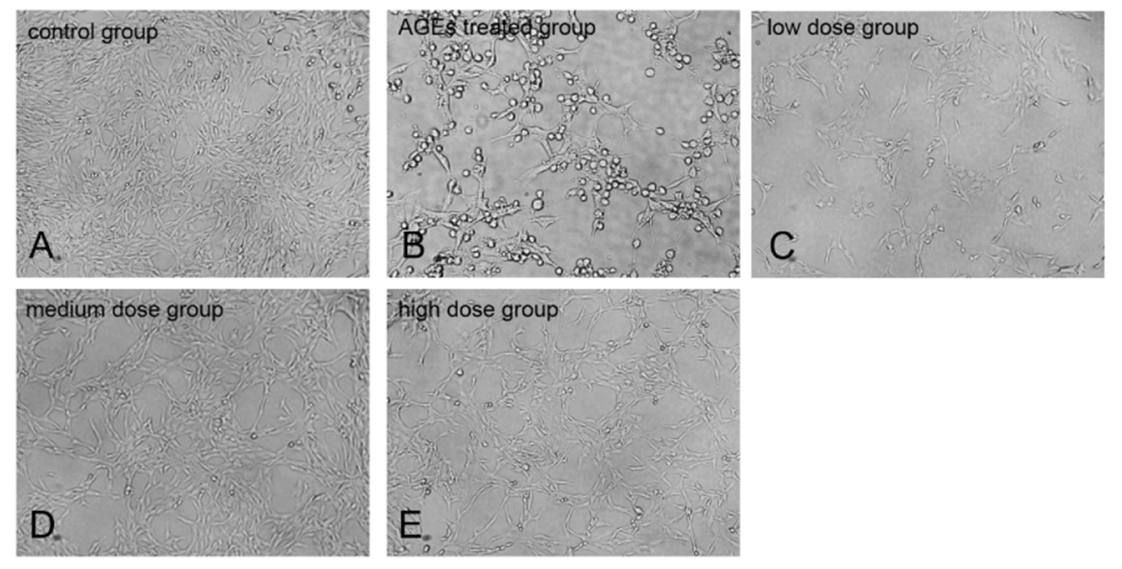

Figure 2. KM treatment protects against AGE-induced GMC injury. Microphotographs of the recovery effect of KM on AGE-induced growth of (A) control group GMCs, (B) cells treated with AGEs $(160 \mu \mathrm{g} / \mathrm{mL})$ for $24 \mathrm{~h}$, and (C-E) cells pre-treated with 10, 20, or $35 \mu \mathrm{M} \mathrm{KM}$ and then stimulated with AGEs $(160 \mu \mathrm{g} / \mathrm{mL})$ for $24 \mathrm{~h}$. The photographs were taken directly from culture plates with an inverted fluorescence microscope (magnification $100 \times$ ).

\subsection{KM Treatment Reduced the Production of ROS in AGE-Treated GMCS}

To determine the effects of KM treatment on the levels of ROS, AGE-treated GMCs were exposed to $2^{\prime}, 7^{\prime}$-dichlorofluorescin diacetate (DCFH-DA), a green fluorescent dye. Treatment with AGEs resulted in an increase in green fluorescence compared to the untreated control group cells (Figure 3A,B), indicating that ROS production increased. Treatment of GMCs with low, medium, and high concentrations of $\mathrm{KM}(10,20$, and $35 \mu \mathrm{M}$, respectively) abated the green fluorescence (Figure 3C-E), indicating that ROS levels were reduced in these cells. These data show that KM treatment results in a decrease in ROS production in AGE-treated GMCs. 

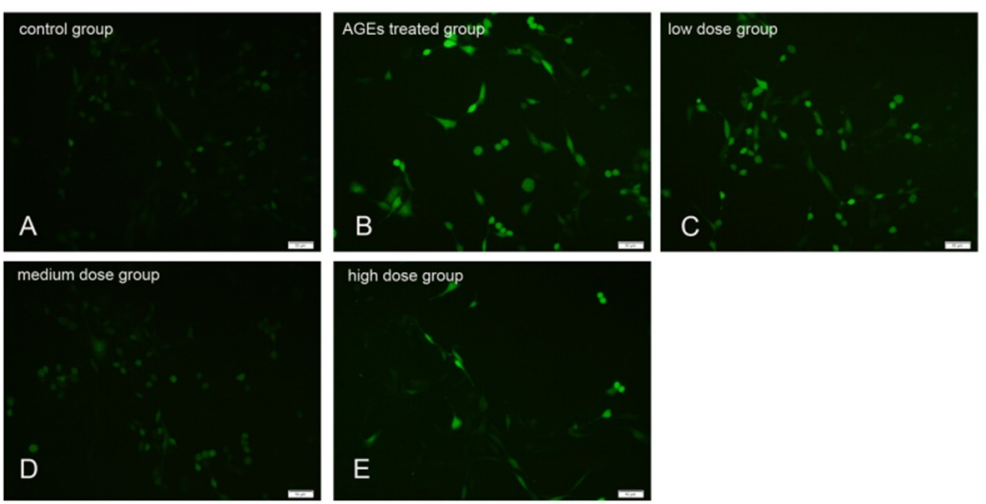

Figure 3. KM treatment reduces the production of ROS in AGE-treated GMCs. Detection of intracellular ROS using DCFH-DA in (A) control group cells, (B) cells treated with AGEs $(160 \mu \mathrm{g} / \mathrm{mL})$ for $24 \mathrm{~h}$, and (C-E) cells pre-treated with 10, 20, or $35 \mu \mathrm{M} \mathrm{KM}$ and then stimulated with AGEs $(160 \mu \mathrm{g} / \mathrm{mL})$ for $24 \mathrm{~h}$. The photographs were taken directly from culture plates with an inverted fluorescence microscope (magnification $50 \times$ ).

\subsection{KM Treatment Improves Mitochondrial Membrane Potential Recovery in AGE-Treated GMCs}

To determine whether KM affects AGE-induced apoptosis in GMCs through the mitochondrial apoptotic pathway, changes in the mitochondrial membrane potential $(\Delta \Psi \mathrm{m})$ were measured. Treatment of GMCs with AGEs resulted in a reduction in the mitochondrial membrane potential from $112.9 \pm 5.0$ to $72.2 \pm 10.1$ (Figure $4 ; p<0.01$ ). When AGE-treated cells were exposed to low, medium, or high concentrations of KM, the mitochondrial membrane potential showed recovery from the AGE control cells, and the membrane potential of the medium concentration KM group was $99.9 \pm 5.1(p<0.01)$. These data show that KM treatment recovers the mitochondrial membrane potential of AGE-treated GMCs, indicating that KM rescues the apoptotic phenotype in these cells.

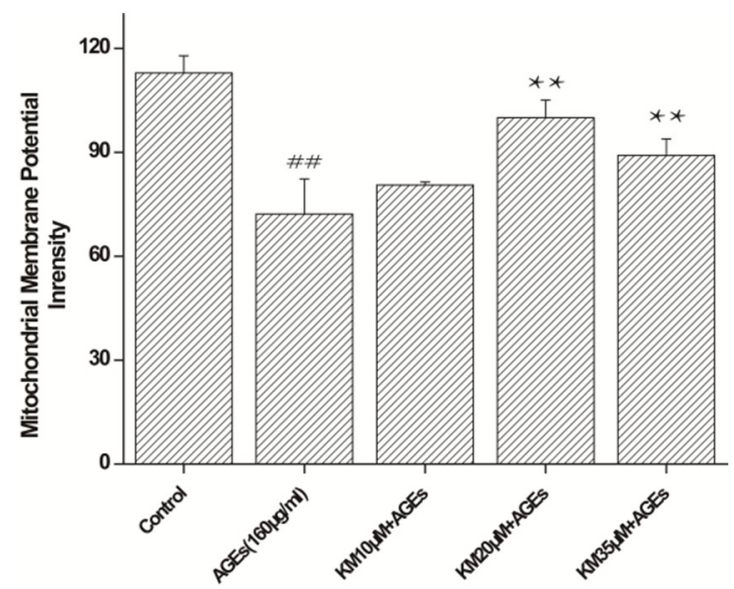

Figure 4. KM treatment improves mitochondrial membrane potential recovery in AGE-treated GMCs. Detection of the changes in mitochondrial membrane potential $(\Delta \Psi \mathrm{m})$ using rhodamine 123. GMCs were pre-treated with 10, 20, or $35 \mu \mathrm{M} \mathrm{KM}$ and then stimulated with AGEs $(160 \mu \mathrm{g} / \mathrm{mL})$ for $24 \mathrm{~h}$. The results are presented as mean \pm SEM. ${ }^{* *} p<0.01$, vs. AGE group; ${ }^{\# \#} p<0.01$, vs. control group.

2.5. KM Treatment Abates the Morphological Changes Induced by Apoptosis and Reduces the Rate of Apoptosis in AGE-Treated GMCS

To detect the effects of KM on the morphology of nuclei in AGE-treated GMCs, Hoechst 33258 staining was used. The nuclei of the untreated control group showed a uniform blue fluorescence, and the color was lightly distributed (Figure 5A). After the addition of AGEs, the staining of the nuclei was dense and fragmented, granular fluorescence was observed, indicating an increase in pyknosis 
(Figure 5B). KM treatment resulted in nuclear staining that was mostly normal, with only an occasional small part remaining densely stained (Figure 5C-E).

To determine how KM treatment affects the apoptotic rate of AGE-treated GMCs, annexin V and PI double staining was used. Figure 6 shows that there were a greater number of GMCs in early apoptosis and late apoptosis and that the apoptosis rate increased upon the addition of AGEs. Treatment with low, medium, or high concentrations of KM resulted in a decrease in the apoptotic rate of AGE-treated GMCs, with the middle dose group having the best results. These data indicate that KM treatment prevents apoptosis-associated morphological changes in the nuclei of and the rate of apoptosis in AGE-treated GMCs.
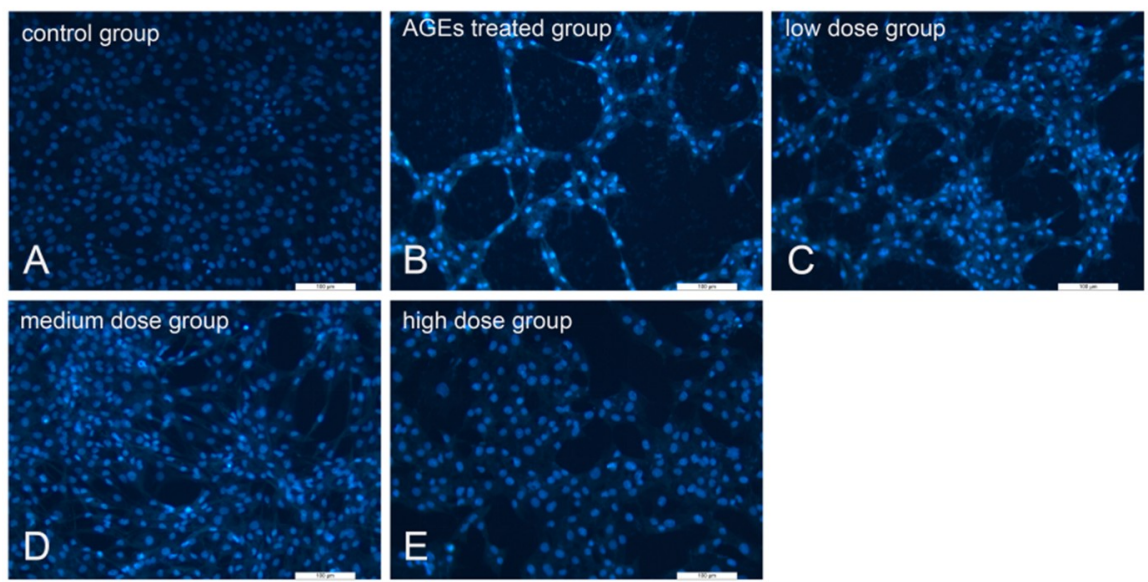

Figure 5. KM treatment prevents apoptosis-related morphological changes in the nuclei of AGE-treated GMCs. Detection of apoptotic morphology using Hoechst 33258 stain in (A) control group cells (B) cells treated with AGEs $(160 \mu \mathrm{g} / \mathrm{mL})$ for $24 \mathrm{~h}$, and (C-E) cells pre-treated with 10, 20, or $35 \mu \mathrm{M} \mathrm{KM}$ and then stimulated with AGEs $(160 \mu \mathrm{g} / \mathrm{mL})$ for $24 \mathrm{~h}$. The photographs were taken directly from culture plates with an inverted fluorescence microscope (magnification $100 \times$ ).
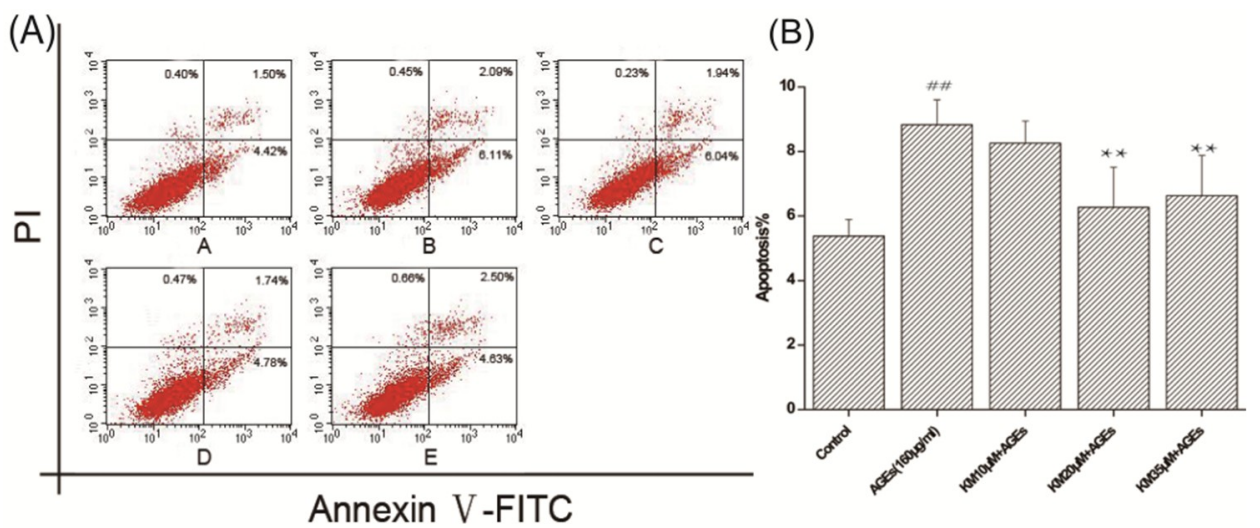

Figure 6. KM treatment reduces the rate of apoptosis in AGE-treated GMCs. Detection of apoptosis rate with annexin V and PI. Cells were stained with annexin V and PI after being treated with AGEs $(160 \mu \mathrm{g} / \mathrm{mL})$ and $\mathrm{KM}(10,20$, and $35 \mu \mathrm{M})$. (A) Percentages of apoptotic cells were determined by flow cytometry. FITC, Fluorescein Isothiocyanate. (B) The results were presented as mean \pm SEM. ** $p<0.01$, vs. AGEs group; ${ }^{\# \#} p<0.01$, vs. control group.

\subsection{KM Modulates the Expression of Both Pro- and Anti-Apoptotic Proteins in GMCs Treated with AGEs}

To determine the effects of KM on the expression of pro- and anti-apoptotic proteins in AGE-treated GMCs, Western blot analysis was used. Upon treatment with AGEs, the level of Bcl-xL was significantly reduced, whereas the level of Bax was significantly increased (Figure 7). Strikingly, treatment with KM resulted in the decrease in the level of Bax. The involvement of cytochrome 
$c$ and caspase activation was also investigated. The levels of cleaved caspases- 9 and -3 increased upon treatment with AGEs, but decreased when KM was added. Furthermore, AGEs induced the expression of RAGE and increased the cleaved form of poly-(adenosine diphosphate-ribose) polymerase (PARP), whereas treatment with KM reduced its expression. These results indicate that KM regulates AGE-induced apoptosis via a mitochondrial apoptotic pathway.

(A)

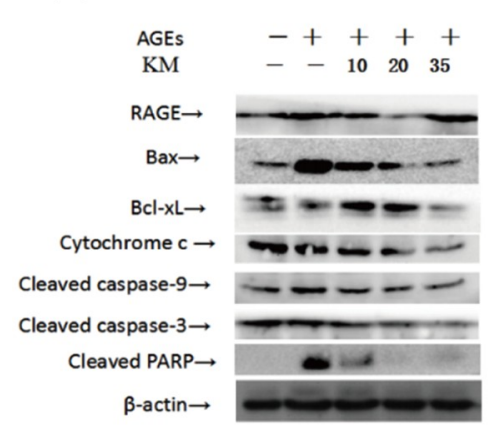

(B)

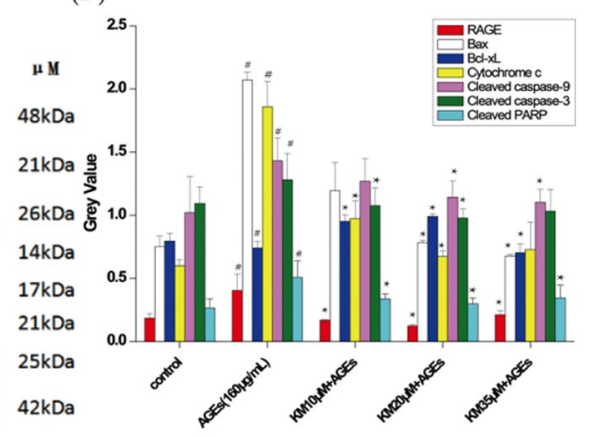

Figure 7. KM modulates the expression of both pro- and anti-apoptotic proteins in GMCs treated with AGEs. Effects of KM on AGE-induced apoptosis-related protein. GMCs were treated with AGEs $(160 \mu \mathrm{g} / \mathrm{mL})$ and $\mathrm{KM}(10,20$, or $35 \mu \mathrm{M})$. (A) The protein levels of RAGE, Bax, Bcl-xL and Cytochrome c, as well as the cleavage of caspases-9, -3 and poly-(ADP-ribose) polymerase (PARP), were examined by Western blot. + means adding reagents, - means not joining (B) The results were presented as mean \pm SEM. ${ }^{*} p<0.05$, vs. AGE group; ${ }^{\#} p<0.05$, vs. control group.

2.7. KM Treatment Enhances Antioxidation and Reduces the Proliferation of the Extracellular Matrix in AGE-Treated GMCs

To determine the degree of the antioxidant activity of KM possesses in AGE-treated cells, analyses using superoxide dismutase (SOD) and malondialdehyde (MDA) kits were combined. SOD activity was reduced in GMCs treated with AGEs $(p<0.05)$ but was increased in response to treatment with KM, particularly by the medium KM dose (Table 1). This finding indicates that KM restores the activity of cells to remove free radicals. Moreover, AGEs increased the MDA content in GMCs $(p<0.05)$, which corresponds to cellular damage of cells. By contrast, KM treatment decreased the MDA content, with the medium dose treatment having the most significant results.

To determine the effect of KM treatment on AGE-induced changes in the extracellular matrix (ECM) of GMCs, Western blot analyses were used. Expression levels of TGF- $\beta 1$ and Collagen IV were increased in AGE-treated GMCs compared to untreated control cells. Treatment with low, medium, or high doses of KM resulted in the decrease in the expression levels of TGF- $\beta 1$ and Collagen IV, consequently reducing the expansion of the ECM (Figure 8).

Table 1. Effects of KM treatment on AGE-induced MDA and SOD content in GMCs.

\begin{tabular}{ccc}
\hline Groups & SOD (U/mL) & MDA (n mol/mL) \\
\hline Control & $1.29 \pm 0.067$ & $0.51 \pm 0.006$ \\
AGEs group & $0.65 \pm 0.006^{*}$ & $0.89 \pm 0.009^{*}$ \\
KM low-dose group & $0.81 \pm 0.007^{*}$ & $0.78 \pm 0.020^{*}$ \\
KM medium-dose group & $0.93 \pm 0.021^{*}$ & $0.58 \pm 0.012^{*}$ \\
KM high-dose group & $0.76 \pm 0.005^{*}$ & $0.69 \pm 0.007^{*}$ \\
\hline & " $p<0.05$, vs. control group; ${ }^{*} p<0.05$, vs. AGEs group.
\end{tabular}


(A)

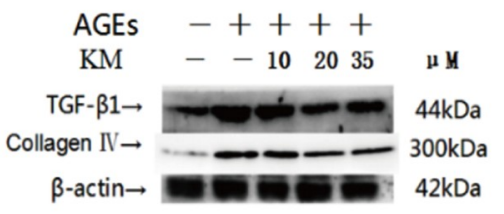

(B)

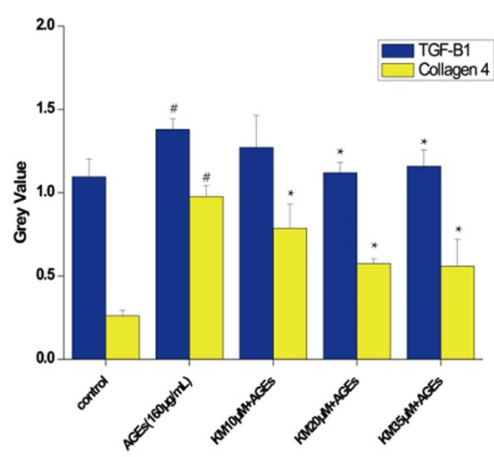

Figure 8. KM treatment reduces the proliferation of the ECM in AGE-treated GMCs. GMCs were treated with AGEs $(160 \mu \mathrm{g} / \mathrm{mL})$ and KM $(10,20$ or $35 \mu \mathrm{M})$. (A) The protein levels of TGF- $\beta 1$ and Collagen IV were examined by Western blot. + means adding reagents, - means not joining $(\mathbf{B})$ The results were presented as mean $\pm \mathrm{SEM}$. ${ }^{*} p<0.05$, vs. AGEs group; ${ }^{\#} p<0.05$, vs. control group.

\section{Discussion}

The early stages of DN are characterized by renal hypertrophy, glomerular hyperfiltration, and microalbuminuria [30]. These changes are related to the subsequent development of glomerular morphological abnormalities and the diagnosis of DN. There is a growing interest in the beneficial roles of KM in the prevention of DM and its complications. As such, we investigated the protective effects of KM against AGE-induced apoptosis and OS in GMCs in vitro.

Extracellular AGEs bind to RAGE, which induces the generation of intracellular ROS and stimulates the production and release of cytokines [31]. TGF- $\beta$ expression is induced by the increase in glucose levels that occur in response to OS induction and AGEs production [32]. TGF- $\beta 1$ augments the deposition of ECM proteins, such as several types of collagens, fibronectin, and laminin at the glomerular level. Therefore, AGEs subsequently induce hypertrophy and fibrosis in GMCs via ROS [33,34]. AGEs play a key role in the development of DM and DM-related complications, as well as promote the deterioration of renal function [35]. Therefore, targeting AGEs could be a suitable therapeutic approach for the prevention of DN.

OS is not only a consequence of AGEs-RAGE activation but also a potent inducer of RAGE expression [36]. Thus, antioxidants should be considered as a treatment strategy for AGEs-RAGE-related diseases such as DN. SOD activity and MDA content are two major indices in the oxidative balance system [37]. KM possesses phenolic hydroxyl groups and natural antioxidative properties, both of which increase SOD activity and decrease MDA content in AGE-induced GMCs. $\mathrm{KM}$ also inhibits the expression of TGF- $\beta 1$ and collagen IV proteins, which may abate the ROS-related damage to GMCs that occurs in DN.

Recent studies have shown that ROS are potent inducers of mitochondrial apoptotic signaling pathways. The maintenance of the mitochondrial membrane potential $(\Delta \psi \mathrm{m})$ is crucial for mitochondrial function. The collapse of $\Delta \Psi \mathrm{m}$ and depletion of ATP are the direct consequences of ROS accumulation in mitochondria, and these events precede the release of cytochrome $\mathrm{c}$ and activation of the caspase protease cascade [38]. We have found that AGEs induce a significant depletion in the $\Delta \Psi \mathrm{m}$ of GMCs, a result that is in agreement with those reported in previous studies [39]. ROS-mediated $\Delta \Psi \mathrm{m}$ depletion and mitochondrial dysfunction are essential for the induction of AGE-induced apoptosis in GMCs. Upon depletion of $\Delta \Psi \mathrm{m}$, there is an AGE-induced activation of caspase-3, which increases the expression of cytochrome $\mathrm{c}$ and its release from the mitochondria.

The release of cytochrome c from mitochondria has been shown to be an almost universal phenomenon during celluar apoptosis. The mitochondria-initiated intrinsic pathway requires the release of cytochrome $\mathrm{c}$ and promotes the caspase-activating apoptosome, a complex that induces the activation of caspase- 9 and initiates the apoptotic caspase cascades [40]. Caspase-3 is regarded as the apoptosis promoter [41]. Caspases have been shown to be activated during apoptosis in 
many cells and play key roles in both the initiation and execution of apoptosis [42]. Mitochondrial apoptosis is regulated mainly by the Bcl-2 family of cell death regulatory molecules, which include the pro-apoptotic Bax and the anti-apoptotic Bcl-2 [43].

Hyperglycemia and OS can trigger an increase in Bax levels, which activates the apoptosis-signaling pathway, including the cleavage of both caspase-3 and caspase-9 [44]. AGEs-induced ROS production in the mitochondria results in cytochrome $c$ release through the regulation of Bax and Bcl-xL [45-47]. Thus, cytochrome c could activate caspase-9 and caspase-3 and stimulate the proteolytic cleavage of PARP, resulting in morphological and membrane potential changes and subsequent mitochondrial and nuclear DNA damage [48,49]. In this study, we found that KM reduces the production of ROS and inhibits the AGE-induced apoptosis of GMCs (Figure 9).

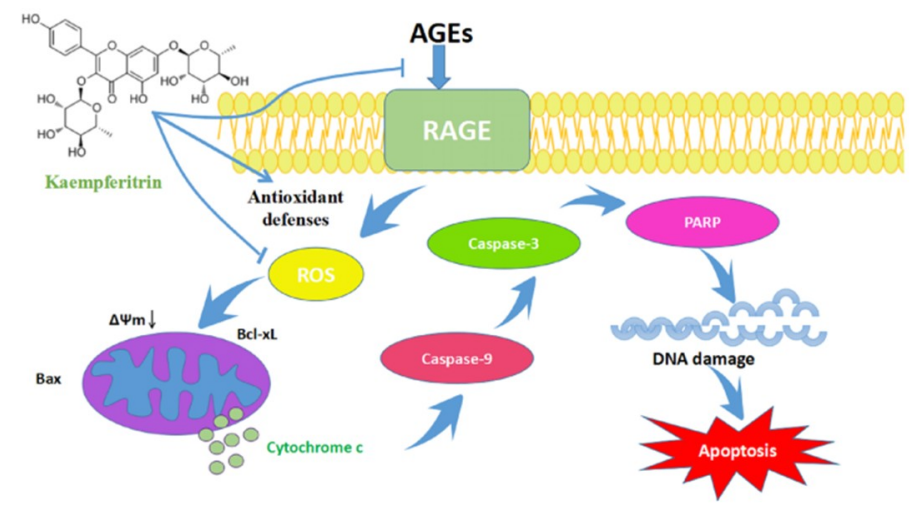

Figure 9. Schematic representation of the biological activities of KM and its effects on the AGE-induced mitochondrial/cytochrome c-mediated apoptosis pathway in GMCs. The thick blue arrows represent the order of action, the thin blue arrows represent the effects of drug intervention, the black arrow represents the decrease in mitochondrial membrane potential, the light green dots represent the release of cytochrome $\mathrm{c}$ in mitochondria, and the different color is for differentiate between different parts and proteins in the cell.

In this study, we explored the effects of KM treatment on AGE-induced injury of GMCs in vitro. The effects of KM on glomerular injury using animal models of DN would greatly enhance the understanding of the protective effects of KM, as well as demonstrate its feasibility as a treatment for DN. Moreover, the in vitro experimental conditions used in our studies did not fully reflect in vivo conditions. Further studies are needed to clarify whether KM treatment could prevent glomerular damage in human DN by blocking the AGEs-RAGE axis and the mitochondrial-mediated apoptotic signaling pathway. Our research contributes to the growing evidence indicating its potential usefulness in treating or managing other diabetic complications. The data reported in this study will facilitate future studies that investigate the in vivo bioavailability and pharmacokinetics of KM, as well as determine the full extent of its protective effects and any toxic side effects.

\section{Materials and Methods}

\subsection{Reagents}

KM (lot NO., 17020911, purity > 98\%) was purchased from Manster Biotechnology Co., Ltd. (Cheng du, China). Dulbecco's modified Eagle's medium (DMEM) and fetal bovine serum (FBS) were purchased from KeyGen Biotechnology Co., Ltd. (Nanjing, China). Bovine serum albumin (BSA), D-glucose and 3-(4,5-Dimethylthiazol-2-yl)-2,5-diphenyltetrazolium bromide (MTT) were obtained from Sigma-Aldrich (St. Louis, MO, USA). Penicillin, streptomycin, rhodamine 123, hoechst 33258, reactive oxygen species assay kit and Bicinchoninicacid (BCA) protein concentration determination kit were purchased from Solarbio Technology Co., Ltd. (Beijing, China). Anti-rat RAGE (ab65965), Bax (ab32503), Bcl-xL (ab32370), Cytochrome C (ab133504), Cleaved-caspase-3 (ab13847), 
Cleaved-caspase-9 (ab25758), Cleaved-PARP (ab32138), TGF- $\beta 1$ (ab92486), Collagen IV (ab6586) and $\beta$-actin (ab8227) were purchased from Abcam Biotechnology Co., Ltd. (Shanghai, China). The annexin $\mathrm{V}$ and PI apoptosis kits were purchased from Invitrogen Thermo Fisher Scientific (Carlsbad, CA, USA). The AGEs Enzyme-linked Immuno Sorbent Assay (ELISA) kit was obtained from R\&D Systems (Minneapolis, MN, USA). The superoxide dismutase (SOD) kit and Malondialdehyde (MDA) kit were purchased from Jiancheng Bioengineering Institute (Nanjing, China).

\subsection{AGEs Preparation}

AGEs-BSA conjugation was prepared by incubating $5 \mathrm{~g}$ bovine serum albumin with $9 \mathrm{~g}$ D-glucose in $10 \mathrm{~mL}$ of phosphate buffer solution (PBS) $(0.2 \mathrm{M}, \mathrm{pH} 7.4)$ for 8 weeks at $37^{\circ} \mathrm{C}$ and the free glucose was dialyzed in $0.01 \mathrm{M}$ PBS (pH 7.4) for $96 \mathrm{~h}$ [50]. The identification of AGEs was determined at excitation $370 \mathrm{~nm}$ and emission $440 \mathrm{~nm}$ in a Gemini EM fluorescence microplate reader (Molecular Devices, Sunnyvale, CA, USA). The content of AGEs was determined using an AGEs ELISA kit [51].

\subsection{Cell Culture}

The rat glomerular mesangial cell (GMC) line was purchased from KeyGen Biotechnology (Nanjing, China) and cultured in Dulbecco's modified Eagle's medium supplemented with 10\% fetal bovine serum, $100 \mathrm{U} / \mathrm{ml}$ penicillin and $100 \mathrm{mg} / \mathrm{ml}$ streptomycin at $37^{\circ} \mathrm{C}$ in $5 \% \mathrm{CO}_{2}$. The medium was replaced every $48 \mathrm{~h}$ until the cells reached $80 \%$ confluency [52].

\subsection{MTT Assay}

GMCs were seeded in 96-well culture plates at $5 \times 10^{3}$ cells $/ \mathrm{mL}$ and then incubated for $24 \mathrm{~h}$. Cells were then treated as follows: (1) Control medium, (2) AGEs (160 $\mu \mathrm{g} / \mathrm{mL})$, (3) different concentrations of KM. After another $24 \mathrm{~h}$ treatment, the culture solution was removed and $5 \mathrm{mg} / \mathrm{mL}$ of the MTT solution was added to each well for $4 \mathrm{~h}$. The medium was then removed and $100 \mu \mathrm{L}$ dimethyl sulfoxide (DMSO) was added to dissolve the formed formazan. The plates were detected at $490 \mathrm{~nm}$ using a microplate reader (Molecular Devices, Sunnyvale, CA, USA).

\subsection{Cellular Morphology Analysis}

GMCs were seeded in six-well culture plates at $10 \times 10^{4}$ cells $/ \mathrm{mL}$ and then incubated for $24 \mathrm{~h}$. Cells were then treated as follows: (1) Control medium, (2) AGEs (160 $\mu \mathrm{g} / \mathrm{mL})$, (3) KM $(10 \mu \mathrm{M})+$ AGEs $(160 \mu \mathrm{g} / \mathrm{mL}),(4) \mathrm{KM}(20 \mu \mathrm{M})+$ AGEs $(160 \mu \mathrm{g} / \mathrm{mL})$, and (5) KM $(35 \mu \mathrm{M})+$ AGEs $(160 \mu \mathrm{g} / \mathrm{mL})$. After $24 \mathrm{~h}$ treatment, the morphological changes were observed under an inverted fluorescence microscope (Olympus, Tokyo, Japan).

\subsection{Determination of SOD and MDA Content}

The SOD and MDA antioxidant indices were measured using the corresponding kits. All procedures were performed in accordance with the manufacturer's instructions. Briefly, GMCs were treated as before, then lyse cells by freeze-thaw cycles, cell supernatants were collected, and SOD and MDA levels were determined according to the manufacturer's instructions.

\subsection{Detection of Intracellular ROS Accumulation}

Intracellular ROS accumulation was monitored using DCFH-DA, which is a specific fluorescent probe [53]. GMCs were seeded in six-well plates and treated as described in Section 4.5. The cells were collected and incubated with $20 \mu \mathrm{M}$ DCFH-DA at $37^{\circ} \mathrm{C}$ for $30 \mathrm{~min}$ in the dark, and then observed under an inverted fluorescence microscope (Olympus, Tokyo, Japan). 


\subsection{Assessment of Mitochondrial Membrane Potential}

The mitochondrial membrane potential $(\Delta \Psi \mathrm{m})$ was determined according previously described methods [54]. Briefly, after treatment according to Section 4.5, GMCs were loaded with $10 \mu \mathrm{M}$ rhodamine 123 and incubated at $37^{\circ} \mathrm{C}$ for $30 \mathrm{~min}$ in the dark. Cells were then harvested, washed, and analyzed with the excitation wavelength at $488 \mathrm{~nm}$ and the emission wavelength at $525 \mathrm{~nm}$ by a microplate reader (Molecular Devices, Sunnyvale, CA, USA).

\subsection{Hoechst 33258 Staining}

Apoptotic morphology was observed using Hoechst 33258 staining [55]. GMCs were treated in groups according to Section 4.5, washed with pre-cooled PBS three times, and incubated in the dark for $10 \mathrm{~min}$ at $37^{\circ} \mathrm{C}$ with $20 \mu \mathrm{M}$ Hoechst 33258. The cells were observed under an inverted fluorescence microscope (Olympus, Tokyo, Japan).

\subsection{Annexin V and PI Double Staining}

The rate of apoptosis was determined by annexin V and PI double staining [56]. After the GMCs were treated in groups according to Section 4.5, the cells were collected and centrifuged at $1000 \mathrm{rpm}$ for $5 \mathrm{~min}$ at $4{ }^{\circ} \mathrm{C}$. Following two PBS washes, the cells were resuspended in $100 \mu \mathrm{L} 1 \mathrm{X}$ binding buffer containing $5 \mu \mathrm{L}$ PI and $5 \mu \mathrm{L}$ FITC-annexin V for 15 min in the dark. Then, $400 \mu \mathrm{L} 1 X$ binding buffer was replenished and the samples were analyzed using flow cytometry (BD Biosciences, Franklin Lake, NV, USA). A total of 10,000 cells were examined per sample, and both early (PI negative and annexin V positive stained cells) and late (PI positive and annexin V positive stained cells) apoptotic cells were analyzed.

\subsection{Subcellular Fractionation for the Detection of Cytochrome C Release}

Subcellular fractionation was used to extract cytochrome c [57]. The cells were collected and washed with cold PBS. $500 \mu \mathrm{L}$ of lysate was ground on a mortar and centrifuged at $1000 \times g$ for $10 \mathrm{~min}$ at $4{ }^{\circ} \mathrm{C}$. The supernatant was collected and centrifuged at $12,000 \times \mathrm{g}$ for $30 \mathrm{~min}$ at $4{ }^{\circ} \mathrm{C}$. The lower layer precipitate was mitochondria, and it was collected and further lysed before analysis by Western blotting.

\subsection{Western Blotting}

The cells were harvested and lysed with radio immunoprecipitation assay (RIPA) buffer (10 mM Tris/ $\mathrm{HCl}, \mathrm{pH} 7.5,150 \mathrm{mM} \mathrm{NaCl}, 1 \%$ Nonidet P-40) containing $1 \mathrm{mM}$ phenylmethanesulfonyl fluoride (PMSF), $1 \mathrm{mM}$ ethylene diamine tetraacetic acid (EDTA), $1 \mu \mathrm{g} / \mathrm{ml}$ pepstatin and $1 \mu \mathrm{g} / \mathrm{mL}$ aprotinin. The protein content was measured using a BCA protein kit. For each sample, $100 \mu \mathrm{g}$ of the lysate were loaded into the sodium dodecyl sulfate polyacrylamide gel electrophoresis (SDS-PAGE) gel and transferred to a polyvinylidene fluoride (PVDF) membrane. The membrane was incubated with $5 \%$ of skim milk powder dissolved in Tris-buffered saline with Tween 20 (TBST) for $1 \mathrm{~h}$. The membrane was then incubated with anti-RAGE, Bax, Bcl-xL, Cytochrome c, Cleaved-caspase-3, Cleaved-caspase-9, PARP, TGF- $\beta 1$, or Collagen IV (1:1000), overnight at $4{ }^{\circ} \mathrm{C}$. The membrane was then washed three times with TBST and incubated with horseradish peroxidase-conjugated anti-rabbit immunoglobulin G (IgG) (1:5000) for $2 \mathrm{~h}$ at room temperature. The signals were detected with the chemiluminescence system. Immunoreactive bands were quantitatively analyzed using Alpha View software (Fluor Chem Q).

\subsection{Statistical Analysis}

All data were presented as mean \pm SEM. The statistical analyses were performed using SPSS 19.0 software (SPSS Inc., Chicago, IL, USA). Analysis of variance (ANOVA) was used to assess the significant differences between multiple groups. Statistical significance was expressed as $p<0.05$. 


\section{Conclusions}

The results of this study revealed that KM increases SOD activity, decreases MDA levels, reduces ROS production, and inhibits the expression of collagen IV and TGF- $\beta 1$. Moreover, these data show that KM suppresses the mitochondrial/cytochrome c-mediated apoptosis pathway by inducing the expression of anti-apoptotic proteins in GMCs in vitro. These findings suggest that KM may be a viable agent for the prevention and treatment of $\mathrm{DN}$.

Author Contributions: W.L. conceived and designed the experiments; W.J., D.L., and M.Z. performed the experiments; R.W. analyzed the data; C.Z. and T.Z. contributed reagents/materials/analysis tools; W.L. and W.J. wrote the paper.

Funding: This research was funded by the National Nature Science Foundation of China (81274049), Natural Science Foundation of Shandong Province (ZR2018MH040), and Staring Foundation for Doctorate Research of Weifang medical university (2017BSQD50).

Acknowledgments: We thank LetPub for its linguistic assistance during the preparation of this manuscript.

Conflicts of Interest: The authors declare no conflict of interest.

\section{References}

1. Husseny, M.W.; Mamdouh, M.; Shaban, S. Adipokines: Potential therapeutic targets for vascular dysfunction in type II diabetes mellitus and obesity. J. Diabetes Res. 2017. [CrossRef] [PubMed]

2. Sidaway, P. Diabetic nephropathy: Heparanase mediates renal injury. Nat. Rev. Nephrol. 2014, 10, 483. [CrossRef] [PubMed]

3. Rossing, P. Diabetic nephropathy: Worldwide epidemic and effects of current treatment on natural history. Curr. Diabetes Rep. 2006, 6, 479-483. [CrossRef]

4. Chu, Y.W.; Lin, H.M.; Wang, J.J. Epidemiology and outcomes of hypoglycemia in patients with advanced diabetic kidney disease on dialysis: A national cohort study. PLoS ONE 2017, 12, e0174601. [CrossRef] [PubMed]

5. Vlassara, H.; Palace, M.R. Diabetes and advanced glycation end products. J. Intern. Med. 2010, $251,87-101$. [CrossRef]

6. Reiniger, N.; Kai, L.; Mccalla, D. Deletion of the receptor for advanced glycation end products reduces glomerulosclerosis and preserves renal function in the diabetic OVE26 mouse. Diabetes 2010, 59, $2043-2054$. [CrossRef] [PubMed]

7. Yamagishi, S.; Nakamura, K.T. Regulation of advanced glycation end product (AGE)-receptor (RAGE) system by PPAR-gamma agonists and its implication in cardiovascular disease. Pharmacol. Res. 2009, 60, 174-178. [CrossRef] [PubMed]

8. Maiese, K. Diabetic stress: New triumphs and challenges to maintain vascular longevity. Expert Rev. Cardiovasc. Ther. 2008, 6, 281-284. [CrossRef] [PubMed]

9. Huang, J.; Matavelli, L.C.; Siragy, H.M. Renal (pro)renin receptor contributes to development of diabetic kidney disease through TGFß1-CTGF signaling cascade. Clin. Exp. Pharmacol. Physiol. 2011, 38, $215-221$. [CrossRef] [PubMed]

10. Martínezpalacián, A.; Castillo, G.D.; Suárezcausado, A. Mouse Hepatic Oval Cells Require Met-Dependent PI3K to Impair TGF- $\beta$-Induced Oxidative Stress and Apoptosis. PLoS ONE 2013, 8, e53108.

11. Fukami, K.; Yamagishi, S.; Ueda, S. Role of AGEs in diabetic nephropathy. Curr. Pharm. Des. 2008, 14, 946-952. [CrossRef] [PubMed]

12. Jefferson, J.A.; Shankland, S.J.; Pichler, R.H. Proteinuria in diabetic kidney disease: A mechanistic viewpoint. Kidney Int. 2008, 74, 22-36. [CrossRef] [PubMed]

13. Stitt, A.W. AGEs and diabetic retinopathy. Investig. Ophthalmol. Vis. Sci. 2010, 51, 4867-4874. [CrossRef] [PubMed]

14. Jorge, A.P.; Horst, H.; De, S.E. Insulinomimetic effects of kaempferitrin on glycaemia and on 14C-glucose uptake in rat soleus muscle. Chem. Biol. Interact. 2004, 149, 89-96. [CrossRef] [PubMed]

15. Li, R.; Jiang, Z.T.; Ma, L. Investigation of antioxidant activities and free radical scavenging of laurel essential oil. China Food Additives 2012, 2, 69-74. 
16. Lin, L.; Ni, B.; Lin, H. Traditional usages, botany, phytochemistry, pharmacology and toxicology of Polygonum multiflorum Thunb: A. review. J. Ethnopharmacol. 2015, 159, 158-183. [CrossRef] [PubMed]

17. Prakash, D.; Sudhandiran, G. Dietary flavonoid fisetin regulates aluminium chloride-induced neuronal apoptosis in cortex and hippocampus of mice brain. J. Nutr. Biochem. 2015, 26, 1527-1539. [CrossRef] [PubMed]

18. Menezes Sousaminto, F.D.; Mattosruela, A.B. Hypoglycemic activity of two Brazilian Bauhinia species: Bauhinia forficata L. and Bauhinia monandra Kurz. Rev. Bras. Farmacogn. 2007, 17, 8-13. [CrossRef]

19. Lino, C.S.; Diógenes, J.P.; Pereira, B.A. Antidiabetic activity of Bauhinia forficata extracts in alloxan-diabetic rats. Biol. Pharm. Bull 2004, 27, 125-127. [CrossRef]

20. Ashafaq, M.; Raza, S.S.; Khan, M.M. Catechin hydrate ameliorates redox imbalance and limits inflammatory response in focal cerebral ischemia. Neurochem. Res. 2012, 37, 1747-1760. [CrossRef] [PubMed]

21. Tzeng, Y.M.; Chen, K.; Rao, Y.K. Kaempferitrin activates the insulin signaling pathway and stimulates secretion of adiponectin in 3T3-L1 adipocytes. Eur. J. Pharmacol. 2009, 607, 27-34. [CrossRef] [PubMed]

22. Abdel-Ghani, N.T.; Shoukry, A.F.; EI Nashar, R.M. Flow injection potentiometric determination of pipazethate hydrochloride. Analyst 2004, 78, 107-113. [CrossRef]

23. Alonso-Castro, A.J.; Ortiz-Sánchez, E.; García-Regalado, A. Kaempferitrin induces apoptosis via intrinsic pathway in HeLa cells and exerts antitumor effects. J. Ethnopharmacol. 2013, 145, 476-489. [CrossRef] [PubMed]

24. Vellosa, J.C.; Regasini, L.O.; Belló, C. Preliminary in vitro and ex vivo evaluation of afzelin, kaempferitrin and pterogynoside action over free radicals and reactive oxygen species. Arch. Pharm. Res. 2015, 38, 1168-1177. [CrossRef] [PubMed]

25. Vishnu-Prasad, C.N.; Suma, M.S.; Banerji, A. Kaempferitrin inhibits GLUT4 translocation and glucose uptake in 3T3-L1 adipocytes. Biochem. Biophys. Res. Commun. 2009, 380, 39-43. [CrossRef] [PubMed]

26. Cazarolli, L.H.; Pereira, D.F.; Kappel, V.D. Insulin signaling: A potential signaling pathway for the stimulatory effect of kaempferitrin on glucose uptake in skeletal muscle. Eur. J. Pharmacol. 2013, 712, 1-7. [CrossRef] [PubMed]

27. Scalbert, A.; Manach, C.; Morand, C. Dietary polyphenols and the prevention of diseases. Crit. Rev. Food Sci. Nutr. 2005, 45, 287-306. [CrossRef] [PubMed]

28. Tang, D.; He, B.; Zheng, Z.G. Inhibitory effects of two major isoflavonoids in Radix Astragali on high glucose-induced mesangial cells proliferation and AGEs-induced endothelial cells apoptosis. Planta Med. 2011, 77, 729-732. [CrossRef] [PubMed]

29. Lee, M.J.; Rao, Y.K.; Chen, K. Andrographolide and 14-deoxy-11,12-didehydroandrographolide from Andrographis paniculata attenuate high glucose-induced fibrosis and apoptosis in murine renal mesangeal cell lines. J. Ethnopharmacol. 2010, 132, 497-505. [CrossRef] [PubMed]

30. Hao, H.H.; Shao, Z.M.; Tang, D.Q. Preventive effects of rutin on the development of experimental diabetic nephropathy in rats. Life Sci. 2012, 91, 959-967. [CrossRef] [PubMed]

31. Yuan, Y.; Zhao, L.; Chen, Y. Advanced glycation end products (AGEs) increase human mesangial foam cell formation by increasing Golgi SCAP glycosylation in vitro. Am. J. Physiol. Renal Physiol. 2011, 301, 236-243. [CrossRef] [PubMed]

32. Kanwar, Y.S.; Wada, J.; Sun, L. Diabetic nephropathy: Mechanisms of renal disease progression. Exp. Biol Med. 2008, 233, 4-11. [CrossRef] [PubMed]

33. Hu, C.; Sun, L.; Xiao, L. Insights into the mechanisms involved in the expression and regulation of extracellular matrix proteins in diabetic nephropathy. Curr. Med. Chem. 2015, 22, 2858-2870. [CrossRef] [PubMed]

34. Fukami, K.; Ueda, S.; Yamagishi, S. AGEs activate mesangial TGF- $\beta$-Smad signaling via an angiotensin II type I receptor interaction. Kidney Int. 2004, 66, 2137-2147. [CrossRef] [PubMed]

35. Navarrogonzález, J.F.; Morafernández, C.; Fuentes, M.M.D. Inflammatory molecules and pathways in the pathogenesis of diabetic nephropathy. Nat. Rev. Nephrol. 2011, 7, 327-340. [CrossRef] [PubMed]

36. Giribabu, N.; Karim, K.; Kilari, E.K. Phyllanthus niruri leaves aqueous extract improves kidney functions, ameliorates kidney oxidative stress, inflammation, fibrosis and apoptosis and enhances kidney cell proliferation in adult male rats with diabetes mellitus. J. Ethnopharmacol. 2017, 205, 123-137. [CrossRef] [PubMed]

37. Xiang, M.; Qian, Z.Y.; Zhou, C.H. Crocetin inhibits leukocyte adherence to vascular endothelial cells induced by AGEs. J. Ethnopharmacol. 2006, 107, 25-31. [CrossRef] [PubMed]

38. Poupel, F.; Aghaei, M.; Movahedian, A. Dihydroartemisinin induces apoptosis in human bladder cancer cell lines through reactive oxygen species, mitochondrial membrane potential, and cytochrome c pathway. Int. J. Prev. Med. 2017, 8, 78. [PubMed] 
39. Lu, M.; Sun, L.; Zhou, J. Dihydroartemisinin induces apoptosis in colorectal cancer cells through the mitochondria-dependent pathway. Tumor Biol. 2014, 35, 5307-5314. [CrossRef] [PubMed]

40. Sun, S.Y.; Hail, N.; Lotan, R. Apoptosis as a Novel Target for Cancer Chemoprevention. J. Natl. Cancer Inst. 2004, 96, 662-672. [CrossRef] [PubMed]

41. Lin, C.F.; Lo, Y.H.; Hsieh, M.C. Cytotoxicities, cell cycle and caspase evaluations of 1,6-diaryl-3(Z.)-hexen-1,5-diynes, 2-(6-aryl-3(Z.)-hexen-1,5-diynyl) anilines and their derivatives. Bioorg. Med. Chem. 2005, 13, 3565-3575. [CrossRef] [PubMed]

42. Liu, Z.; Hou, Y.; Dong, M. PA-MSHA inhibits proliferation and induces apoptosis through the up-regulation and activation of caspases in the human breast cancer cell lines. J. Cell Biochem. 2010, 108, 195-206. [CrossRef] [PubMed]

43. Granadoserrano, A.B.; Martín, M.A.; Bravo, L. Quercetin induces apoptosis via caspase activation, regulation of Bcl-2, and inhibition of PI-3-kinase/Akt and ERK pathways in a human hepatoma cell line (HepG2). J. Nutr. 2006, 136, 2715-2721. [CrossRef] [PubMed]

44. Song, X.; Wang, B.; Lin, S. Astaxanthin inhibits apoptosis in alveolar epithelial cells type II in vivo and in vitro through the ROS-dependent mitochondrial signalling pathway. J. Cell Mol. Med. 2015, 18, 2198-2212. [CrossRef] [PubMed]

45. Fuchs, Y.; Steller, H. Live to die another way: Modes of programmed cell death and the signals emanating from dying cells. Nat. Rev. Mol. Cell Biol. 2015, 16, 329-344. [CrossRef] [PubMed]

46. Desagher, S.; Osen-Sand, A.; Nichols, A. Bid-Induced conformational change of Bax is responsible for mitochondrial cytochrome c release during apoptosis. J. Cell Biol. 1999, 144, 891-901. [CrossRef] [PubMed]

47. Chipuk, J.E.; Green, D.R. How do BCL-2 proteins induce mitochondrial outer membrane permeabilization. Trends Cell Biol. 2008, 18, 157-164. [CrossRef] [PubMed]

48. Li, P.; Nijhawan, D.; Budihardjo, I.; Srinivasula, S.M.; Ahmad, M.; Alnemri, E.S. Cytochrome c and dATP-dependent formation of Apaf-1/caspase-9 complex initiates an apoptotic protease cascade. Cell 1997, 91, 479-489. [CrossRef]

49. Wang, I.K.; Lin-Shiau, S.Y.; Lin, J.K. Induction of apoptosis by apigenin and related flavonoids through cytochrome c release and activation of caspase-9 and caspase-3 in leukaemia HL-60 cells. Eur. J. Cancer 1999, 35, 1517-1525. [CrossRef]

50. Chen, Y.; Du, X.; Zhou, Y. Paeoniflorin protects HUVECs from AGE-BSA-induced injury via an autophagic pathway by acting on the RAGE. Int. J. Clin. Exp. Pathol. 2015, 8, 53-62. [PubMed]

51. Zhang, M.; Feng, L.; Gu, J. The attenuation of moutan cortex on oxidative stress for renal injury in AGEs-induced mesangial cell dysfunction and streptozotocin-induced diabetic nephropathy rats. Oxid. Med. Cell Longev. 2015, 2014, 463815. [CrossRef] [PubMed]

52. Zhang, K.; Meng, X.; Li, D. Angiotensin(1-7) attenuates the progression of streptozotocin-induced diabetic renal injury better than angiotensin receptor blockade. Kidney Int. 2015, 87, 359-369. [CrossRef] [PubMed]

53. Zhang, W.; Song, J.; Zhang, Y. Intermittent high glucose-induced oxidative stress modulates retinal pigmented epithelial cell autophagy and promotes cell survival via increased HMGB1. BMC Ophthalmol. 2018, 18, 192. [CrossRef] [PubMed]

54. Sureda, F.X.; Escubedo, E.; Gabriel, C. Mitochondrial membrane potential measurement in rat cerebellar neurons by flow cytometry. Cytometry 2015, 28, 74-80. [CrossRef]

55. Li, W.; Yan, M.H.; Liu, Y.; Liu, Z.; Wang, Z.; Chen, C.; Zhang, J.; Sun, Y.S. Ginsenoside Rg5 ameliorates cisplatin-induced nephrotoxicity in mice through inhibition of inflammation, oxidative stress, and apoptosis. Nutrients 2016, 8, 566. [CrossRef] [PubMed]

56. Sharma, S.; Singh, R.L.; Kakkar, P. Modulation of Bax/Bcl-2 and caspases by probiotics during acetaminophen induced apoptosis in primary hepatocytes. Food Chem. Toxicol. 2011, 49, 770-779. [CrossRef] [PubMed]

57. Chen, H.M.; Wu, Y.C.; Chia, Y.C. Gallic acid, a major component of Toona sinensis leaf extracts, contains a ROS-mediated anti-cancer activity in human prostate cancer cells. Cancer Lett. 2009, 286, 161-171. [CrossRef] [PubMed]

(C) 2018 by the authors. Licensee MDPI, Basel, Switzerland. This article is an open access article distributed under the terms and conditions of the Creative Commons Attribution (CC BY) license (http://creativecommons.org/licenses/by/4.0/). 